\title{
GPU facilitated unsupervised visual feature acquisition
}

\author{
Blake Lemoine*, Anthony Maida \\ From Twenty First Annual Computational Neuroscience Meeting: CNS*2012 \\ Decatur, GA, USA. 21-26 July 2012
}

This paper examines the effects of parallelizing an unsupervised feature acquisition algorithm using GeneralPurpose computing on Graphics Processing Units (GPGPU). Previous work in feature learning has established the effectiveness of hierarchical learning methods that refine large quantities of elementary features into small numbers of intermediate-level features. Some of the most effective of these methods alternate between template matching layers that combine features and subsampling layers that perform feature scale and/or position invariance. This approach is inspired by the mammalian ventral visual pathway. One such approach that produces highly informative features is a spiking convolutional network trained with STDP-based learning [1]. When using a serial implementation, however, this approach is costly in time and consequently is difficult to scale to a large library of internally complex features. The present paper demonstrates that GPGPU parallelism can be leveraged to overcome the scaling limitations of the serial version. Highly informative features can then be generated in large quantities. By scaling the number of features learned the maximum complexity of learnable classes is significantly increased.

Published: 16 July 2012

\section{Reference}

1. Masquelier $T$, Thorpe $\mathrm{S}$ : Unsupervised learning of visual features through spike timing dependent plasticity. PLOS Computational Biology 2007, 3.

doi:10.1186/1471-2202-13-S1-P87

Cite this article as: Lemoine and Maida: GPU facilitated unsupervised visual feature acquisition. BMC Neuroscience 2012 13(Suppl 1):P87.
Submit your next manuscript to BioMed Central and take full advantage of:

- Convenient online submission

- Thorough peer review

- No space constraints or color figure charges

- Immediate publication on acceptance

- Inclusion in PubMed, CAS, Scopus and Google Scholar

- Research which is freely available for redistribution
() Biomed Central 